\title{
On the Turbulence in a Viscous Heat-Conducting Gas
}

\author{
Sergey Voronkov \\ Institute of Engineering Sciences \\ Pskov State University \\ Pskov, Russia \\ vorss60@yandex.ru \\ Alexander Dementiev \\ Institute of Engineering Sciences \\ Pskov State University \\ Pskov, Russia \\ damix01@yandex.ru \\ Tatiana Bugaeva \\ Institute of Engineering Sciences \\ Pskov State University \\ Pskov, Russia \\ tanya.bugaeva.69@mail.ru
}

\author{
Pavel Safronov \\ Institute of Engineering Sciences \\ Pskov State University \\ Pskov, Russia \\ safisfppi@list.ru \\ Olga Frolova \\ Institute of Engineering Sciences \\ Pskov State University \\ Pskov, Russia \\ _backof2@mail.ru
}

\begin{abstract}
The problem of the emergence of turbulence is one of the unsolved problems of physics and technology of the 20th century. It is noted that in order to understand the emergence of turbulence in a viscous heat-conducting gas, it is necessary to take into account the compressibility of the medium. A definition of turbulence in a viscous heatconducting gas is given, which is a cyclically repeating process of the emergence and decay of coherent vortex structures described by a vector wave equation. The decay of vortex structures is accompanied by an explosive, asymptotic increase in pressure pulsations, which triggers a new cycle of turbulence generation. The emergence and decay of coherent vortex structures in the boundary layer on a plate and in a round jet is considered.
\end{abstract}

Key words - turbulence, viscous heat-conducting gas, coherent vortex structures, vector wave equation, boundary layer, round jet.

\section{INTRODUCTION}

The problem of the emergence of turbulence is one of the unsolved problems of physics and technology of the 20th century. Turbulent flow of liquid and gas is the main one for engineering equipment. Laminar modes are implemented extremely rarely, at low flow rates. Energetically, laminar regimes are more preferable, since the pressure losses in them are proportional to the first power of the flow rate, in contrast to the turbulent flow regime, where the pressure losses are proportional to the flow rate squared. It is traditionally believed [1] that the process of turbulence emergence can be considered in the approximation of the incompressibility of the medium, which greatly simplifies the mathematical formulation of the problem. But as shown in [2], the assumption that the medium is incompressible, which is valid for liquids, is unreasonably extrapolated to a viscous heat-conducting gas. The assumption about the incompressibility of the medium assumes that the divergence of the flow rate is zero $\operatorname{div} \boldsymbol{V}=0$. In [2], as a result of a computational experiment, it was found that the divergence of velocity in a viscous heat-conducting gas at the leading edge of the plate undergoes a sharp jump with a negative sign and its value is $\operatorname{div} \boldsymbol{V}=-800$. This result casts doubt on the validity of the assumption about the incompressibility of the medium when turbulence emergences in a viscous heat-conducting gas. To understand the emergence of turbulence in a viscous heat-conducting gas, it is 
necessary to take into account the compressibility of the medium.

\section{TURBULENCE IN A VISCOUS HEAT-CONDUCTING GAS}

Taking into account the compressibility of the medium and the dissipation of energy in a viscous heatconducting gas during the emergence of turbulence made it possible to obtain a vector wave equation describing the emergence and decay of coherent vortex structures [3]

$\frac{\partial^{2} \boldsymbol{\omega}}{\partial t^{2}}=\left(a_{s}^{2}+\frac{4}{3}(k-1) v \operatorname{div} \boldsymbol{V}\right)$ graddiv $\omega$,

where $\boldsymbol{\omega}$ - is the circular frequency of the vortex tube; $a_{s}$ - is adiabatic and isentropic value of the speed of sound; $\boldsymbol{V}$ - is the vector of the pulsating gas velocity with projections $u, v, w$ on the axis of the Cartesian coordinate system $x, y, z$ respectively; $v-$ is coefficient of kinematic viscosity; $k-$ is the adiabatic index; $t$ - is time.

Coherent vortex structures emergences and decays during the transition to turbulence - Fig. 1

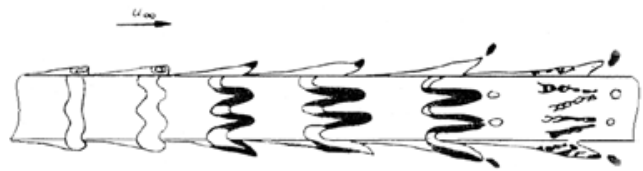

Fig. 1. Visualization of the process of emergence and decay of coherent vortex structures on a body of revolution. Figure from [4]. Quoted from [5]

The emergence and decay of coherent vortex structures occurs not only at the stage of transition, but also in a developed turbulent flow. The resulting equation (1) allows us to give the following definition of turbulence in a viscous heat-conducting gas [3]:

Turbulence in a viscous heat-conducting gas is a cyclically repeating process of the emergence and decay of coherent vortex structures described by a vector wave equation. The decay of vortex structures is accompanied by an explosive, asymptotic increase in pressure pulsation, which triggers a new cycle of turbulence generation.

An expression for the pressure pulsation arising during the decay of coherent vortex structures was obtained in [6]

$\Delta p=4 \mu(k-1) \frac{\omega_{0}^{2} t_{0}^{2}}{t_{0}-t}$,

where $\omega_{0}-$ is the circular frequency of the vortex tube before the beginning of decay, $t_{0}-$ is the total decay time of the vortex tube, $\mu-$ is the coefficient of dynamic viscosity.
Let us give a graph of the change in pressure pulsation that occurs during the decay of vortex structures - Fig. 2.

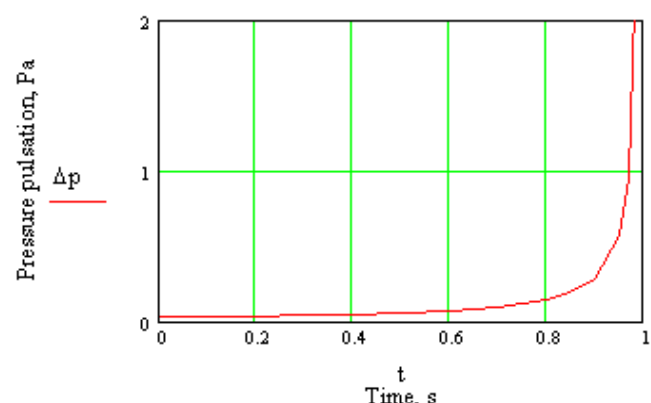

Fig. 2. Pressure pulsations calculated by the formula (2). When calculating it was taken: $\mu=1,82 \cdot 10^{-5} \mathrm{~Pa} \cdot \mathrm{s} ; k=1,4$; $\omega_{0}=10 \pi \mathrm{rad} / \mathrm{s} ; t_{0}=1 \mathrm{~s}$. Figure from [6]

Under the influence of pressure pulsation (2) at the place of the decay of coherent vortex structures, the formation of Emmons turbulent spots occurs - Fig. 3

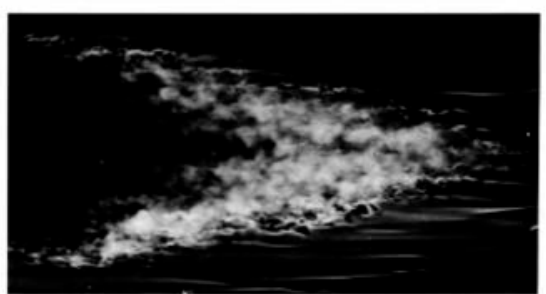

Fig. 3. Emmons turbulent spot. Reynolds number $R e=400000$. Visualization is carried out using smoke in the air, illuminated by a flash. Photo by R.E. Falco. Figure from [7]

Turbulent spots, propagating downstream, merge and form a developed turbulent flow.

\section{COHERENT VORTEX STRUCTURES IN THE BOUNDARY LAER ON THE PLATE}

Consider the emergence of vortex tubes in a natural transition on a plate - Fig. 4
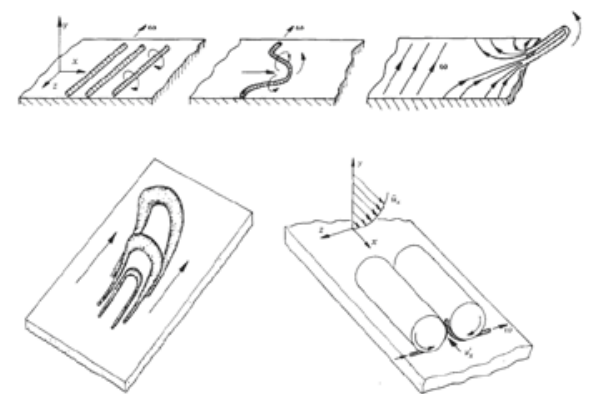

Fig. 4. The appearance of two-dimensional vortices and their decay into three-dimensional structures in a natural transition. Figure from [8]. Quoted from [9]

Circular frequency is related to speed circulation 
$\omega=\frac{1}{2} \operatorname{rot} V$

Considering that [10]

$\operatorname{divrot} \boldsymbol{V}=0$,

Substituting (3) and (4) in (1), we obtain

$\frac{\partial^{2} \boldsymbol{\omega}}{\partial t^{2}}=0$

We write the vector equation (5) in projections on the axes of the Cartesian coordinate system $x, y, z$

$$
\begin{aligned}
\frac{\partial^{2} \omega_{x}}{\partial t^{2}} & =0, \\
\frac{\partial^{2} \omega_{y}}{\partial t^{2}} & =0, \\
\frac{\partial^{2} \omega_{z}}{\partial t^{2}} & =0 .
\end{aligned}
$$

The solutions to equations (6-8) will be written

$\omega_{x}=C_{1} t+C_{2}$,

$\omega_{y}=C_{3} t+C_{4}$,

$\omega_{z}=C_{5} t+C_{6}$,

where $C_{1}, C_{2}, C_{3}, C_{4}, C_{5}, C_{6}$ - are constants.

In a natural transition, vortex tubes arise - twodimensional vortices - Fig. 4, perpendicular to the main direction of flow. Consequently, constants $C_{1}, C_{2}, C_{3}, C_{4}$ will be equal to zero

$C_{1}, C_{2}, C_{3}, C_{4}=0$

Let us consider a special case of the solution when the initial value of the circular frequency of the vortex tube does not depend on time and $C_{5}=0$.

Then the initial value of the vortex tube will be determined by a constant, which can be found as

$C_{6}=\frac{1}{2}\left(\frac{\partial v}{\partial x}-\frac{\partial u}{\partial y}\right)$

that is, the initial value of the circular frequency of the vortex tube is determined by the derivatives of the pulsation components of the velocities $u$ and $v$

$\omega_{z}=\frac{1}{2}\left(\frac{\partial v}{\partial x}-\frac{\partial u}{\partial y}\right)$

A further change in the circular frequency of the vortex tube $\omega_{z}$ is described by the vector wave equation (1).
Let's write it in projections on the axes of the Cartesian coordinate system $x, y, z$

$$
\begin{aligned}
& \frac{\partial^{2} \omega_{x}}{\partial t^{2}}=\left(a_{s}^{2}+\frac{4}{3}(k-1) v \operatorname{div} \boldsymbol{V}\right)\left[\frac{\partial^{2} \omega_{x}}{\partial x^{2}}+\frac{\partial^{2} \omega_{y}}{\partial y \partial x}+\frac{\partial^{2} \omega_{z}}{\partial z \partial x}\right], \\
& \frac{\partial^{2} \omega_{y}}{\partial t^{2}}=\left(a_{s}^{2}+\frac{4}{3}(k-1) v \operatorname{div} \boldsymbol{V}\right)\left[\frac{\partial^{2} \omega_{x}}{\partial x \partial y}+\frac{\partial^{2} \omega_{y}}{\partial y^{2}}+\frac{\partial^{2} \omega_{z}}{\partial z \partial y}\right], \\
& \frac{\partial^{2} \omega_{z}}{\partial t^{2}}=\left(a_{s}^{2}+\frac{4}{3}(k-1) v \operatorname{div} \boldsymbol{V}\right)\left[\frac{\partial^{2} \omega_{x}}{\partial x \partial z}+\frac{\partial^{2} \omega_{y}}{\partial y \partial z}+\frac{\partial^{2} \omega_{z}}{\partial z^{2}}\right] .
\end{aligned}
$$

An analysis of equations (15-17) shows that a vortex tube with circular frequency $\omega_{z}$ arising in the boundary layer will generate coherent vortex structures with frequencies $\omega_{x}$ and $\omega_{y}$ and will itself be deformed under the action of these vortex structures. Indeed, in equations (15) and (16), the spatial derivatives of frequency $\omega_{z}$ appeared on the right side, which will generate vortex tubes with frequencies $\omega_{x}$ and $\omega_{y}$. Also, according to equation (17), the initially two-dimensional vortex tube and its circular frequency $\omega_{z}$ will deform and change, which is reflected in Fig. 4. Coherent vortex structures disintegrate and generate turbulent spots. The generation of turbulent spots occurs under the influence of pressure pulsations (2)

\section{COHERENT VORTEX STRUCTURES IN A ROUND JET}

Let us consider the emergence and decay of coherent vortex structures in a round jet. During the natural transition in a round jet, ring vortices arise - vortex Kelvin - Helmholtz rings and streaky structures that are longitudinal vortices - Fig. 5 .

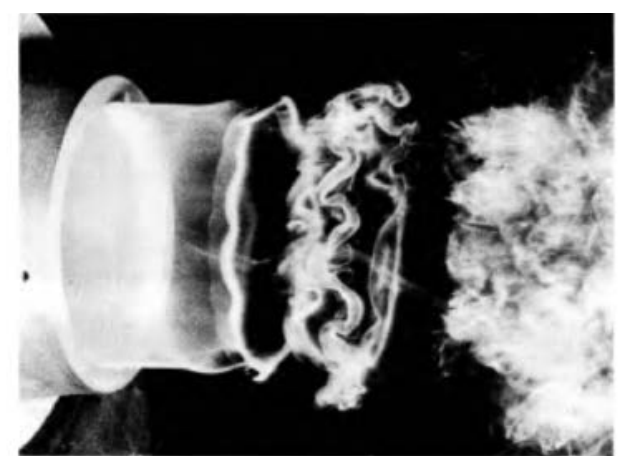

Fig. 5. Natural transition in a round jet. Photo by R. Wille, A Michalke. Figure from [7]

Streaky structures, as noted in [11], appear in jets directly at the exit from flat and round nozzles.

It is more convenient to consider physical processes for a circular axisymmetric jet in cylindrical coordinates $r, \varepsilon, z$. The $\mathrm{z}$ axis is compatible with the jet axis. We write the vector equation (5) in projections on the axes of the cylindrical coordinate system 
$\frac{\partial^{2} \omega_{r}}{\partial t^{2}}=0$

$\frac{\partial^{2} \omega_{\varepsilon}}{\partial t^{2}}=0$

$\frac{\partial^{2} \omega_{z}}{\partial t^{2}}=0$

The solutions of equations (18-20) will be written

$\omega_{r}=C_{1} t+C_{2}$,

$\omega_{\varepsilon}=C_{3} t+C_{4}$,

$\omega_{z}=C_{5} t+C_{6}$,

where $C_{1}, C_{2}, C_{3}, C_{4}, C_{5}, C_{6}-$ are constants.

In the natural transition of a round jet, ring vortices $-\omega_{\varepsilon}$ and streaky structures - longitudinal vortices $-\omega_{z}$ appear at the nozzle exit. Consequently, constants $C_{1}, C_{2}$ will be equal to zero

$C_{1}, C_{2}=0$.

Let us consider a particular case of solutions when the initial values of the circular frequencies of the vortex tubes do not depend on time and $C_{3}, C_{5}=0$. Then the initial values of the vortex tubes will be determined by constants $C_{4}, C_{6}$, which can be found as

$C_{4}=\frac{1}{2}\left(\frac{\partial V_{r}}{\partial z}-\frac{\partial V_{z}}{\partial r}\right)$,

$C_{6}=\frac{1}{2 r}\left(\frac{\partial\left(r V_{\varepsilon}\right)}{\partial r}-\frac{\partial V_{r}}{\partial \varepsilon}\right)$,

where $V_{r}, V_{\varepsilon}, V_{z}$ - are the projections of the pulsating gas velocities on the axis of the cylindrical coordinate system $r, \varepsilon, z$, respectively.

That is, the initial values of the circular frequencies of the vortex tubes are determined by the derivatives of the pulsation velocity components

$\omega_{\varepsilon}=\frac{1}{2}\left(\frac{\partial V_{r}}{\partial z}-\frac{\partial V_{z}}{\partial r}\right)$,

$\omega_{z}=\frac{1}{2 r}\left(\frac{\partial\left(r V_{\varepsilon}\right)}{\partial r}-\frac{\partial V_{r}}{\partial \varepsilon}\right)$.

Further variation of the circular frequencies is described by the vector wave equation (1).

As noted in [11]: "An important aspect concerning streaky structures is their role in the process of turbulization of axisymmetric flows, for example, in a round jet. Such structures arise in jets directly at the exit from flat and round nozzles. When two-dimensional vortex rings of Kelvin - Helmholtz interact with streaky structures, their three-dimensional distortion occurs with the formation of characteristic azimuthal $\Lambda-$ or $\Omega-$ shaped ejections (Fig. 6)."

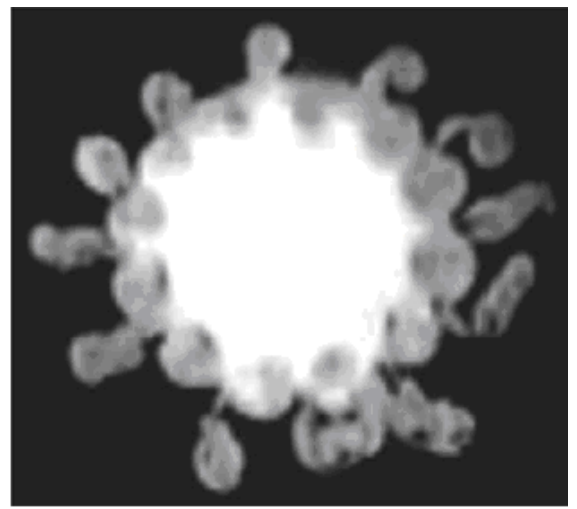

Fig. 6. Visualization of the cross-section of a round jet during the interaction of an ring vortex with streaky structures and the formation of azimuthal $\Lambda$-structures. Figure from [11]

The interaction of streaky structures with ring vortices leads to their decay with the formation of turbulent spots.

Coherent vortex structures appear in both laminar and turbulent jets.

In [12], the mechanism of the emergence and development of coherent vortex structures in laminar and turbulent round jets was investigated. The authors of [12] came to the following conclusions: "It has been shown that the mechanism of the emergence and development of coherent structures in laminar and turbulent jets is identical in general terms and is associated with the emergence and development of coherent structures such as Kelvin - Helmholtz ring vortices, streaky structures, and their interaction in both types of flow."

\section{CONCLUSION}

To understand the emergence of turbulence in a viscous heat-conducting gas, it is necessary to take into account the compressibility of the medium.

The presented vector wave equation for the circular frequency of a vortex tube describes the emergence and decay of coherent vortex structures in the boundary layer on a plate and in a round axisymmetric jet.

It is noted that coherent vortex structures are present not only at the transition stage, but also in the developed turbulent flow of a viscous gas.

It is shown that turbulence in a viscous heatconducting gas is a cyclically repeating process of the emergence and decay of coherent vortex structures described by the vector wave equation $\frac{\partial^{2} \boldsymbol{\omega}}{\partial t^{2}}=\left(a_{s}^{2}+\frac{4}{3}(k-1) \nu \operatorname{div} \boldsymbol{V}\right)$ graddiv $\boldsymbol{\omega}$. The decay of vortex structures is accompanied by an explosive, asymptotic increase in pressure pulsation $\Delta p=4 \mu(k-1) \frac{\omega_{0}^{2} t_{0}^{2}}{t_{0}-t}$, which triggers a new cycle of turbulence generation. 


\section{REFERENCES}

[1] Schlichting G. Theory of the boundary layer. Moscow: Nauka, 1974, 712 p.

[2] Voronkov S. S. On the connection of Tollmin-Schlichting waves with acoustic waves. Scientific journal "Noise Theory and Practice", - access Mode. - URL: http://www.noisetp.com/ru/issues/journal-issues-archive/vol-6-no4/, Volume 6 №4 (IV, 2020). Pp. 42-48.

[3] Voronkov S.S. On the emergence and decay of coherent vortex structures in a viscous gas. Electronic journal "Technical acoustics", http://www.ejta.org/2021 №1.

[4] Knapp C.F., Roache P.J. A combined visual and hot-wire, anemometerinvestigation of boundary layer transition. - AIAA J., 1968, v. 6, № 1, p. 29-36.

[5] Kachanov Y.S., Kozlov V.V., Levchenko V.Ya. Arising Turbulence in a Boundary Layer. Novosibirsk: Nauka, 151 pages, 1982.
[6] Voronkov S. S. On the mechanism of emergence turbulent Emmons spots. Electronic journal "Technical Acoustics", http://www.ejta.org/2020 №1

[7] Van Dyke M. Album of fluid and gas flows. - M.: Mir. 1986. $184 \mathrm{p}$.

[8] Davidson, P. A. Turbulence: an introduction for scientists and engineers. Oxford, UK: Oxford University Press, 2004. - 680 p.

[9] Garbaruk A.V. Transition to turbulence. Lecture 2. - S-P . $\begin{array}{lllll}\text { SPbSPU, } & 2019 & . & & \end{array}$ https://cfd.spbstu.ru/agarbaruk/turb_models/Term8_Lec02_transit ion.pdf

[10] Loitsyansky L.G. Mechanics of fluid and gas. Ed. 5th. - M.: Nauka, 1978. - 736 p.

[11] Boyko A.V., Gorev V.N., Kozlov V.V. Transition to turbulence in boundary layers: Success and prospects. NSU Bulletin. Series: Physics. 2006. Volume 1, Issue 2. P. 21-28.

[12] Kozlov G.V., Litvinenko Yu. A., Grek G.R., Sorokin A.M. On the mechanism of the emergence and development of coherent structures in laminar and turbulent round jets. NSU Bulletin. Series: Physics. 2008. Volume 3, Issue 1. P. 12-22. 